\title{
Triangulations and the Hajós Conjecture
}

\author{
Bojan Mohar* \\ Department of Mathematics, \\ University of Ljubljana, \\ 1000 Ljubljana, Slovenia \\ bojan.mohar@fmf.uni-lj.si
}

Submitted: Apr 18, 2005; Accepted: Sep 1, 2005; Published: Sep 14, 2005

Mathematics Subject Classifications: 05C10, 05C15

\begin{abstract}
The Hajós Conjecture was disproved in 1979 by Catlin. Recently, Thomassen showed that there are many ways that Hajós conjecture can go wrong. On the other hand, he observed that locally planar graphs and triangulations of the projective plane and the torus satisfy Hajós Conjecture, and he conjectured that the same holds for arbitrary triangulations of closed surfaces. In this note we disprove the conjecture and show that there are different reasons why the Hajós Conjecture fails also for triangulations.
\end{abstract}

\section{Introduction}

Hajós conjecture claims that every graph whose chromatic number is at least $k$ contains a subdivision of $K_{k}$, the complete graph of order $k$. The conjecture has been proved for $k \leq 4$ by Dirac [3], while for $k=5$, it yields a strengthening of the Four Color Theorem, which is still open. The conjecture was disproved for all $k \geq 7$ by Catlin [2]. Soon after that, Erdős and Fajtlowicz [4] proved that the conjecture is false for almost all graphs.

Recently, Thomassen [11] revived the interest in Hajós conjecture by showing that there is a great variety of reasons why Hajós conjecture can be wrong. At the end of this interesting work, Thomassen observed that the Hajós conjecture could be true to some limited extent. Maybe it holds in the setting under whose influence it was originally formulated (related to the Four Color Conjecture). For instance, it holds for graphs embedded in any fixed surface with sufficiently large edge-width (i.e., when all noncontractible cycles are long). Therefore, it seems plausible to propose:

* Supported in part by the Ministry of Education, Science and Sport of Slovenia, Research Project J1-0502-0101 and Research Program P1-0297. 
Conjecture 1.1 (Thomassen [11]) Every graph that triangulates some surface satisfies Hajós conjecture.

Every $n$-vertex triangulation of a nonplanar surface has at least $3 n-3$ edges. Therefore, it contains a subdivision of $K_{5}$ by a theorem of Mader [6]. In particular, possible counterexamples to Conjecture 1.1 must have chromatic number at least 6 . Thomassen [11] used a known list of 6- and 7-critical graphs on the projective plane and the torus [10] to prove that Conjecture 1.1 holds for triangulations on these two surfaces.

In this note we provide counterexamples to Conjecture 1.1. Additionally, we give some reasons showing that, in certain sense, almost all graphs should be close to some counterexamples.

\section{A small counterexample}

Let $\tilde{H}$ be the graph with vertex set

$$
V=\left\{v_{i, j} \mid 0 \leq i \leq 4,0 \leq j \leq 2\right\} \cup\left\{w_{i} \mid 0 \leq i \leq 4\right\}
$$

in which distinct vertices $v_{i, j}$ and $v_{k, l}$ are adjacent if and only if $k \in\{i-1, i, i+1\}$ (where $i-1$ and $i+1$ are taken modulo 5), and $w_{i}$ is adjacent to all vertices $v_{i, j}$ and $v_{i+1, j}$, $0 \leq j \leq 2$. In other words, $\tilde{H}$ is composed of 5 copies $Q_{i}(0 \leq i \leq 4)$ of the graph $K_{7}$ with vertices $C_{i}^{-}=\left\{v_{i, 0}, v_{i, 1}, v_{i, 2}\right\}, C_{i}^{+}=\left\{v_{i+1,0}, v_{i+1,1}, v_{i+1,2}\right\}$, and $w_{i}$, and $C_{i}^{+} \subset Q_{i}$ is identified with $C_{i+1}^{-} \subset Q_{i+1}$ for $0 \leq i \leq 4$ (indices modulo 5).

The graph $\tilde{H}$ is a counterexample to Conjecture 1.1:

Theorem 2.1 The graph $\tilde{H}$ triangulates the orientable surface of genus 6 and the nonorientable surface of genus 12 . Its chromatic number is 8 and it does not contain a subdivision of $K_{8}$.

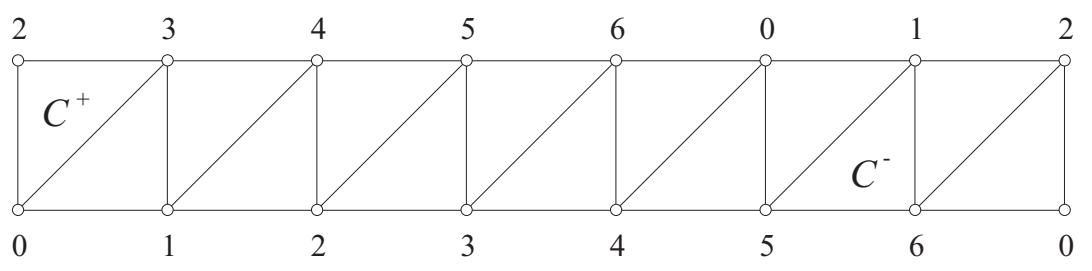

Figure 1: $K_{7}$ on the torus

Proof. The graph $K_{7}$ triangulates the torus. See Figure 1, where the edge on the left is identified with the rightmost one, and the two cycles of the resulting cylinder are then identified as shown by the labels. Consider 5 such triangulations using $Q_{0}, \ldots, Q_{4}$. We may assume that the triangles $C_{i}^{+}$and $C_{i}^{-}$of each $Q_{i}$ are facial; suppose that they correspond to the triangles that are labeled 023 and 156 in Figure 1. By identifying $C_{i}^{+}$ 
with $C_{i+1}^{-}$for $i=0,1,2,3$, we obtain the connected sum of five tori. Finally, by identifying $C_{4}^{+}$with $C_{0}^{-}$, the orientable surface of genus 6 is obtained. Clearly, the resulting graph is isomorphic to $\tilde{H}$ and triangulates the surface. Instead of the orientable surface we can get a nonorientable surface of the same Euler characteristic by taking for $C_{0}^{-}$in $Q_{0}$ the triangle 165 instead of 156.

Let $H$ be the subgraph of $\tilde{H}$ obtained by removing vertices $w_{0}, \ldots, w_{4}$. Clearly, no three vertices of $H$ are independent. Thus, $\chi(H) \geq\lceil|V(H)| / 2\rceil=8$. Also, it is easy to find an 8-coloring of $H$, and any such coloring extends to $\tilde{H}$ since the degrees of the removed vertices $w_{i}$ are equal to 6 . Consequently, $\chi(\tilde{H})=\chi(H)=8$.

Let us now prove that $\tilde{H}$ does not contain a subdivision of $K_{8}$. Assume, by reductio ad absurdum, that $L \subseteq \tilde{H}$ is such a subgraph. Vertices of degree 7 in $L$ are branch vertices; vertices of degree 2 subdivide edges of $K_{8}$. Since $\operatorname{deg}_{\tilde{H}}\left(w_{i}\right)=6$, no $w_{i}$ is a branch vertex. Since the neighbors of $w_{i}$ form a complete subgraph, we may also assume that $w_{i}$ does not subdivide an edge in $L$ for $i=0, \ldots, 4$. In particular, we conclude that $L \subseteq H$.

Let $B \subseteq V(L)$ be the set of branch vertices and let $s$ be the number of vertices of degree 2 in $L$. Clearly, $|B|=8$ and $s \leq|V(H) \backslash B|=7$. Let $B_{i}=B \cap C_{i}^{+}$and $b_{i}=\left|B_{i}\right|$ for $0 \leq i \leq 4$. We may assume that $b_{0}=\max _{i} b_{i}$ and that, subject to this assumption, $b_{0}+b_{1}$ is maximum. Then it is easy to see that $\left(b_{0}, b_{1}\right) \in\{(3,3),(3,2),(3,1),(2,2)\}$. We let $b=b_{0}+b_{1}$.

There are $8-b$ branch vertices that need to be joined to $b$ branch vertices in $B_{0} \cup B_{1}$. Each such vertex $x \in B_{2} \cup B_{3} \cup B_{4}$ is linked to all vertices in $B_{0} \cup B_{1}$; some of the subdivided edges must contain two or three vertices of degree 2 . It is easy to see that for every such $x$, at least four vertices of degree 2 are needed, with two possible exceptions in the cases when $b_{0}=b_{1}=2$ or $b_{0}=3, b_{1}=1$. In any case, we conclude that $s \geq 8$, and this contradiction completes the proof.

\section{$3 \quad$ Paley graphs}

Let $q=p^{r}$ be a prime power, where $p \equiv 1(\bmod 4)$. The Paley graph $\mathbf{P}_{q}$ is the Cayley graph of the additive group of the finite field $G F(q)$ generated by all squares, i.e., $V\left(\mathbf{P}_{q}\right)=$ $G F(q)$ and two distinct vertices $x, y$ are adjacent if and only if $x-y=z^{2}$ for some $z \in G F(q)$. Since $p \equiv 1(\bmod 4), x-y$ is a square if and only if $y-x$ is a square, and this assures that $\mathbf{P}_{q}$ is a graph and not a digraph.

Paley graphs have a number of intriguing properties. First of all, they are highly symmetric. Secondly, they are self-complementary. On the other hand, they resemble random graphs a lot. One of their properties which makes them similar to random graphs is the following result of Thomason [9]; see also [1, p. 363].

Theorem 3.1 Let $A$ be a set of vertices of the Paley graph $\mathbf{P}_{q}$. Let $a=|A|$, and let $e(A)$ be the number of edges in the induced subgraph $\mathbf{P}_{q}(A)$. Then

$$
\left|e(A)-\frac{1}{2}\left(\begin{array}{l}
a \\
2
\end{array}\right)\right| \leq \frac{a(q-a)}{4 \sqrt{q}} .
$$


This theorem implies that the maximum clique in $\mathbf{P}_{q}$ has at most $\sqrt{q}$ vertices. Equality is attained if $q$ is an even power of a prime. But if $q$ is a prime, it seems that the maximum cliques in $\mathbf{P}_{q}$ have far smaller orders. It is believed that their orders are at most polylogarithmic, possibly $o\left(\log ^{2} q\right)$ or even smaller. See [1] for some discussion on this problem.

Let $z$ be a generator of the multiplicative group $G F(q)^{*}$. It is known that this group is cyclic, so $z^{0}, z^{2}, z^{4}, \ldots, z^{q-3}$ are precisely all squares. For each vertex $x \in V\left(\mathbf{P}_{q}\right)$, its neighbors are $x+z^{0}, x+z^{2}, x+z^{4}, \ldots, x+z^{q-3}$. This (cyclic) sequence defines a local rotation around $x$, i.e., a clockwise cyclic order of edges incident with $x$. The collection of all such local rotations defines an orientable embedding $\Pi_{q}$ of $\mathbf{P}_{q}$ which is known as the Paley map. It has the following properties (see [12]):

Theorem 3.2 Suppose that $q=p^{r}$, where $p \equiv 1(\bmod 8)$ is a prime. Then the Paley map $\Pi_{q}$ is self-dual - the geometric dual graph $\mathbf{P}_{q}^{*}$ is isomorphic to $\mathbf{P}_{q}$. Its genus is equal to $\left(q^{2}-9 q+8\right) / 8$. It has $q$ faces, each of which has length $(q-1) / 2$ and is bounded by a cycle of $\mathbf{P}_{q}$.

We refer to [12] for more details concerning this interesting map.

From now on we assume that $q \equiv 1(\bmod 8)$. Let $T_{q}$ be the triangulation of the orientable surface of genus $\left(q^{2}-9 q+8\right) / 8$ obtained from the Paley map $\Pi_{q}$ by adding a new vertex into each face and joining it to all vertices on the boundary of that face. Let $V_{q}=V\left(\mathbf{P}_{q}\right)$ and let $V_{q}^{*}$ be the added vertices. Then $\left|V_{q}\right|=\left|V_{q}^{*}\right|=q$.

Theorem 3.3 Suppose that $q=p^{r}$, where $p \equiv 1(\bmod 8)$ is a prime. The triangulation $T_{q}$ contains no subdivision of the complete graph of order $\geq \lambda \sqrt{q}$, where $\lambda=\frac{1}{4}(2+\sqrt{198})<$ 4.0178.

Proof. Let $s=\sqrt{q}$ and $k=\lceil\lambda s\rceil$. Suppose that $T_{q}$ contains a subdivision $K$ of $K_{k}$. Let $B_{0} \subseteq V(K)$ be the set of branch vertices, and let $b=\left|B_{0} \cap V_{q}\right|$ and $b^{*}=\left|B_{0} \cap V_{q}^{*}\right|$. Clearly, $b+b^{*}=k$.

Let us count the number of subdivided edges in $K$. Since no two vertices in $V_{q}^{*}$ are adjacent, $B_{0} \cap V_{q}^{*}$ gives rise to $\left(\begin{array}{c}b^{*} \\ 2\end{array}\right)$ subdivided edges. Concerning the set $B_{0} \cap V_{q}$, Theorem 3.1 shows that this set gives rise to at least $\frac{1}{2}\left(\begin{array}{l}b \\ 2\end{array}\right)-\frac{b(q-b)}{4 s}$ subdivided edges. Since the total number of vertices of $T_{q}$ is equal to $2 q$, we see that:

$$
k+\left(\begin{array}{c}
b^{*} \\
2
\end{array}\right)+\frac{1}{2}\left(\begin{array}{l}
b \\
2
\end{array}\right)-\frac{b(q-b)}{4 s} \leq 2 q .
$$

Let $b^{*}=\alpha^{*} s$ and $b=\alpha s$. Then (1) expands to the following condition

$$
s\left(2 \alpha^{* 2}+\alpha^{2}-\alpha-8\right)+2 \alpha^{*}+3 \alpha+\alpha^{2} \leq 0 .
$$

Let $\Lambda=\alpha^{*}+\alpha$. Then (2) implies that

$$
2(\Lambda-\alpha)^{2}+\alpha^{2}-\alpha-8<0 .
$$


By considering (3) as a quadratic inequality in $\alpha$, its discriminant is $97+8 \Lambda-8 \Lambda^{2}$. Therefore, (3) has no solutions if

$$
8 \Lambda^{2}-8 \Lambda-97 \geq 0
$$

Since $\Lambda s=k=\lceil\lambda s\rceil \geq \lambda s$, we have $\Lambda \geq \lambda=\frac{1}{4}(2+\sqrt{198})$. This implies (4) and proves that (3) has no solution. This contradiction completes the proof.

Theorem 3.3 now implies:

Theorem 3.4 Suppose that $q=p^{r}$, where $p \equiv 1(\bmod 8)$ is a prime. If the chromatic number of $\mathbf{P}_{q}$ is at least $\lambda \sqrt{q}$, where $\lambda=\frac{1}{4}(2+\sqrt{198})$, then the triangulation $T_{q}$ fails to satisfy the Hajós Conjecture.

In the above theorem, it suffices to assume that $\chi\left(T_{q}\right) \geq \lambda \sqrt{q}$. But since the vertex set $V_{q}^{*}$ is independent in $T_{q}, \chi\left(T_{q}\right) \leq \chi\left(\mathbf{P}_{q}\right)+1$. Therefore, we find it more pleasing to use the natural value $\chi\left(\mathbf{P}_{q}\right)$.

The independence numbers $\alpha\left(\mathbf{P}_{q}\right)$ of Paley graphs of prime order $q$ less than 7000 were computed by Shearer [8]. Using these calculations and estimate the chromatic number by $\chi\left(\mathbf{P}_{q}\right) \geq\left\lceil q / \alpha\left(\mathbf{P}_{q}\right)\right\rceil$, one finds out that Theorem 3.4 can be applied for many values of $q$ (and when $q$ gets large, almost all of them are good). All such primes $q \leq 7000$ with $q \equiv 1(\bmod 8)$ are collected in Table 1 .

As stated after Theorem 3.1, it is believed that for every prime $q, \omega\left(\mathbf{P}_{q}\right)=o(\sqrt{q})$. If true, this yields infinitely many cases where Theorem 3.4 can be applied.

\section{Conclusions}

We have proved that there is a variety of reasons why a triangulation of some surface may fail to satisfy the Hajós Conjecture. In this sense, this note can be viewed as an echo to the stimulating work of Thomassen [11]. As noted in that paper, graphs embedded in a surface $\Sigma$ with sufficiently large edge-width satisfy the Hajós Conjecture. Recall that the edge-width of a graph embedded in a nonplanar surface is the length of a shortest noncontractible cycle. In the above observation of Thomassen, the required width depends on $\Sigma$. However, the following strengthening may be true:

Conjecture 4.1 There is an absolute constant $w_{0}$ such that every graph which can be embedded in some surface with edge-width at least $w_{0}$ satisfies the Hajós Conjecture.

Kühn and Osthus [5] proved that graphs whose girth is at least 186 satisfy the Hajós conjecture. This excludes the most obvious possibility of counterexamples to Conjecture 4.1. (Note that the edge-width of a graph of girth $g$ is at least $g$.)

We do not dare to estimate what the best possible value for $w_{0}$ may be. However, if we restrict ourselves to triangulations, no counterexamples of edge-width 4 are known. 


\begin{tabular}{|c|c|c|c|c|c|c|c|}
\hline$q$ & $\alpha\left(\mathbf{P}_{q}\right)$ & $\left\lceil q / \alpha\left(\mathbf{P}_{q}\right)\right\rceil$ & $\overline{\lambda \lambda \sqrt{q}}$ & $q$ & $\alpha\left(\mathbf{P}_{q}\right)$ & $\overline{\left\lceil q / \alpha\left(\mathbf{P}_{q}\right)\right.}$ & $\lceil\lambda \sqrt{q}$ \\
\hline 3697 & 15 & 247 & 245 & 5953 & 17 & 351 & 310 \\
\hline 4217 & 15 & 282 & 261 & 6073 & 19 & 320 & 314 \\
\hline 4441 & 15 & 297 & 268 & 6089 & 17 & 359 & 314 \\
\hline 4457 & 15 & 298 & 269 & 6113 & 19 & 322 & 315 \\
\hline 4649 & 17 & 274 & 274 & 6121 & 18 & 341 & 315 \\
\hline 4673 & 17 & 275 & 275 & 6217 & 19 & 328 & 317 \\
\hline 4721 & 17 & 278 & 277 & 6257 & 19 & 330 & 318 \\
\hline 4729 & 17 & 279 & 277 & 6329 & 19 & 334 & 320 \\
\hline 4793 & 16 & 300 & 279 & 6337 & 17 & 373 & 320 \\
\hline 4817 & 17 & 284 & 279 & 6353 & 17 & 374 & 321 \\
\hline 4937 & 17 & 291 & 283 & 6361 & 18 & 354 & 321 \\
\hline 4969 & 17 & 293 & 284 & 6449 & 19 & 340 & 323 \\
\hline 4993 & 17 & 294 & 284 & 6473 & 19 & 341 & 324 \\
\hline 5081 & 17 & 299 & 287 & 6481 & 17 & 382 & 324 \\
\hline 5153 & 17 & 304 & 289 & 6529 & 17 & 385 & 325 \\
\hline 5233 & 17 & 308 & 291 & 6553 & 19 & 345 & 326 \\
\hline 5281 & 17 & 311 & 292 & 6569 & 19 & 346 & 326 \\
\hline 5297 & 17 & 312 & 293 & 6577 & 19 & 347 & 326 \\
\hline 5393 & 17 & 318 & 296 & 6673 & 19 & 352 & 329 \\
\hline 5441 & 17 & 321 & 297 & 6689 & 19 & 353 & 329 \\
\hline 5449 & 17 & 321 & 297 & 6737 & 18 & 375 & 330 \\
\hline 5569 & 18 & 310 & 300 & 6761 & 20 & 339 & 331 \\
\hline 5657 & 18 & 315 & 303 & 6793 & 19 & 358 & 332 \\
\hline 5737 & 17 & 338 & 305 & 6833 & 19 & 360 & 333 \\
\hline 5801 & 17 & 342 & 307 & 6841 & 17 & 403 & 333 \\
\hline 5849 & 17 & 345 & 308 & 6857 & 17 & 404 & 333 \\
\hline 5857 & 17 & 345 & 308 & 6961 & 19 & 367 & 336 \\
\hline 5881 & 19 & 310 & 309 & 6977 & 18 & 388 & 336 \\
\hline 5897 & 17 & 347 & 309 & & & & \\
\hline
\end{tabular}

Table 1: Some good values of $q$

Problem 4.2 Does every triangulation without noncontractible triangles satisfy the Hajós Conjecture?

For a graph $H$ and a positive integer $t$, let $\tilde{H}_{t}$ be the graph obtained from the union of $H$ and the clique $K_{t}$ by adding all edges between them. Clearly, the Hajós Conjecture holds for $H$ if and only if it holds for $\tilde{H}_{t}$.

Let $v$ and $e(\tilde{v}$ and $\tilde{e})$ be the number of vertices and edges of $H$ (and $\tilde{H}_{t}$ ), respectively. Clearly, $\tilde{v}=v+t$ and $\tilde{e}=e+\left(\begin{array}{l}t \\ 2\end{array}\right)+v t$. If $\tilde{H}_{t}$ triangulates some surface, then Euler's formula implies that $\tilde{e}$ is divisible by 3 . This holds if and only if one of the following holds: 
(A) $e \equiv 0(\bmod 3)$ and $t \equiv 0(\bmod 3)$,

(B) $v+e \equiv 0(\bmod 3)$ and $t \equiv 1(\bmod 3)$, or

(C) $e-v \equiv 2(\bmod 3)$ and $t \equiv 2(\bmod 3)$.

Conjecture 4.3 For every graph $H$, there exists a constant $t_{0}$ such that for every $t \geq t_{0}$ for which one of $(\mathrm{A})-(\mathrm{C})$ is satisfied, the graph $\tilde{H}_{t}$ triangulates some surface.

Conjecture 4.3 would imply that for every counterexample $H$ to the Hajós Conjecture for which either $e \equiv 0(\bmod 3), v+e \equiv 0(\bmod 3)$, or $e-v \equiv 2(\bmod 3)$, there are infinitely many triangulations of the form $\tilde{H}_{t}$ which violate the Hajós Conjecture.

Conjecture 4.3 would follow from a solution of a more general open problem which has been raised in [7, Problem 4.4.10]:

Problem 4.4 Is there an $\varepsilon>0$ such that every graph of order $n$, with e edges, where $e$ is divisible by 3 , and with minimum degree at least $(1-\varepsilon) n$ triangulates some surface?

A positive answer to Problem 4.4 would imply that almost one third of random graphs in $\mathcal{G}(n, p)$, where $p>1-\varepsilon$, would be graphs of some triangulations. As shown by Erdős and Fajtlowicz [4], almost all of these graphs would also be counterexamples to Conjecture 1.1.

\section{References}

[1] B. Bollobás, Random graphs, $2^{\text {nd }}$ ed., Cambridge University Press, Cambridge, 2001.

[2] P. Catlin, Hajós' graph-coloring conjecture: variations and counterexamples, J. Combin. Theory, Ser. B 26 (1979) 268-274.

[3] G. A. Dirac, A property of 4-chromatic graphs and some remarks on critical graphs, J. London Math. Soc. 27 (1952) 85-92.

[4] P. Erdős, S. Fajtlowicz, On the conjecture of Hajós, Combinatorica 1 (1981) 141-143.

[5] D. Kühn, D. Osthus, Topological minors in graphs of large girth, J. Combin. Theory, Ser. B 86 (2002) 364-380.

[6] W. Mader, $3 n-5$ edges do force a subdivision of $K_{5}$, Combinatorica 18 (1998) 569-595.

[7] B. Mohar and C. Thomassen, Graphs on Surfaces, Johns Hopkins University Press, Baltimore, 2001.

[8] J. B. Shearer, http://www.research.ibm.com/people/s/shearer/indpal.html

[9] A. G. Thomason, Paley graphs and Weil's theorem, Talk at the British Combinatorial Conference, Southampton, 1983 (unpublished).

[10] C. Thomassen, Five-coloring graphs on the torus, J. Combin. Theory Ser. B 62 (1994) 11-33.

[11] C. Thomassen, Some remarks on Hajós' conjecture, J. Combin. Theory, Ser. B 93 (2005) 95-105.

[12] A. T. White, Graphs of groups on surfaces. Interactions and models, North-Holland, Amsterdam, 2001. 